\title{
Current Chemistry Letters
}

\section{A survey on application of mesoporous materials in chemistry}

\author{
S. J. Sadjadi ${ }^{a *}$ and M. Reza Naimi-Jamal ${ }^{\mathrm{b}}$
}

${ }^{a}$ Department of Industrial Engineering, Iran University of Science and Technology, Tehran, Iran ${ }^{b}$ Department of Chemistry, Iran University of Science and Technology, Tehran, Iran

\begin{tabular}{l}
\hline C H R O N I C L E \\
\hline Article history: \\
Received January 5, 2019 \\
Received in revised form \\
February 20, 2019 \\
Accepted February 20, 2019 \\
Available online \\
February 22, 2019 \\
\hline Keywords: \\
Chemistry \\
Scientometrics \\
Bibliography \\
Mesoporous materials
\end{tabular}

\section{Introduction}

Mesoporous materials are substances whose pores maintain diameters between 2 and $50 \mathrm{~nm}$, according to IUPAC nomenclature. ${ }^{1}$ According to IUPAC, microporous materials contain pores smaller than $2 \mathrm{~nm}$ in diameter, while macroporous materials are with pores bigger than $50 \mathrm{~nm}$ in diameter. Most mesoporous materials include different kinds of silica and alumina with similarly-sized mesopores. There are several evidences of mesoporous oxides of niobium, tantalum, titanium, zirconium, cerium and tin but the flagship of mesoporous materials is mesoporous carbon with direct implementation in energy storage facilities. ${ }^{2}$ This paper presents a comprehensive scientometrics on the existing trend on mesoporous materials in chemistry. The study uses Web of Science database as the primary source of value added articles and performs different methods for detecting highly cited articles, most active countries, etc.

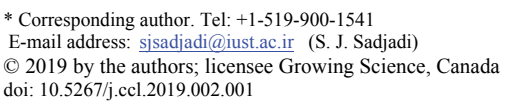

\begin{abstract}
Mesoporous materials are substances whose pores maintain diameters between 2 and $50 \mathrm{~nm}$, according to IUPAC nomenclature. This paper presents a comprehensive scientometrics on the as the primary source of value added articles and performs different methods for detecting highly cited articles, most active countries, etc. The search of articles using Web of Science was accomplished with two keywords "Mesoporous materials" and "Chemistry". In Web of Science, there were about 800 articles related to these keywords over the period 1900-2019. Then the articles were arranged according to the citation order in non-increasing order, and among them, we found about 200 highly cited articles. According to our survey, green chemistry, water, heterogeneous catalysis and aqueous-solution build a structure on heterogeneous catalysts set the corner of other structure of the study. The survey also indicates that there were three clusters associated with mesoporous materials in chemistry. In the first cluster silica appears to be the most important word followed by molecular-sieves and MCM41. Organic-group is the most important word in the second cluster followed by hybrid materials. In cluster 3, nanoparticles appears to be the most important word followed by functionalization. In our study, absorption, nanoparticles and drug-delivery are detected as the emerging keywords and future studies could be concentrated on these subjects.
\end{abstract}




\section{The bibliographic study}

In this study, we have used Web of Science database as the primary source of gathering data and the collected information are used through Biblioshiny tool available in R-software package for processing the data.

\subsection{The themes in reviewed articles}

The search of articles on the Scopus site was accomplished with two keywords "Mesoporous materials" and "Chemistry". In Web of Science, there were about 750 articles related to these keywords. Then the articles were arranged according to the citation non-increasing order, and among them, we have reported about 200 highly cited articles. Table 1 demonstrates some of the most cited references associated with the application of mesoporous materials in chemistry. As we can observe from the results of Table 1, mesoporous materials, molecular-sieves and silica are three well-recognized keywords used in the literature. Fig. 1 shows the most important words used overtimes. Moreover, Fig. 1 demonstrates the trend and various words used in the literature.

Table 1 The most popular keywords used in studies associated with mesoporous materials

\begin{tabular}{|c|c|c|c|}
\hline Words & Occurrences & Words & Occurrences \\
\hline chemistry & 195 & heterogeneous catalysis & 15 \\
\hline mesoporous materials & 141 & polymers & 15 \\
\hline molecular-sieves & 112 & size & 15 \\
\hline silica & 109 & templates & 15 \\
\hline adsorption & 67 & aqueous-solution & 14 \\
\hline nanoparticles & 60 & aqueous-solutions & 14 \\
\hline MCM-41 & 50 & guest molecules & 14 \\
\hline catalysts & 43 & inorganic hybrid materials & 14 \\
\hline thin-films & 39 & self-assembled monolayers & 14 \\
\hline Design & 38 & spectroscopy & 14 \\
\hline surface & 35 & surfaces & 14 \\
\hline complexes & 33 & triblock & 14 \\
\hline ordered mesoporous materials & 33 & immobilization & 13 \\
\hline drug-delivery & 32 & release & 13 \\
\hline controlled-release & 31 & responsive controlled-release & 13 \\
\hline functionalization & 31 & system & 13 \\
\hline frameworks & 30 & template & 13 \\
\hline SBA-15 & 30 & activated carbon & 12 \\
\hline organic groups & 29 & carbon & 12 \\
\hline mesoporous molecular-sieves & 28 & derivatives & 12 \\
\hline surface-chemistry & 26 & gel & 12 \\
\hline click chemistry & 25 & molecules & 12 \\
\hline hybrid materials & 24 & pore structure & 12 \\
\hline mesoporous silica & 24 & sieves & 12 \\
\hline mechanism & 23 & surface organometallic chemistry & 12 \\
\hline oxidation & 22 & temperature & 12 \\
\hline surfactant & 22 & zeolites & 12 \\
\hline Acid & 21 & catalyst & 11 \\
\hline pore-size & 21 & efficient & 11 \\
\hline channel walls & 20 & epoxidation & 11 \\
\hline copolymer & 20 & gold nanoparticles & 11 \\
\hline Films & 20 & in-vitro & 11 \\
\hline heterogeneous catalysts & 20 & metal-oxides & 11 \\
\hline oxide & 20 & organic-synthesis & 11 \\
\hline green chemistry & 19 & porous silicon & 11 \\
\hline silica nanoparticles & 19 & selective oxidation & 11 \\
\hline catalysis & 18 & supramolecular chemistry & 11 \\
\hline porous materials & 18 & surface-area & 11 \\
\hline separation & 18 & systems & 11 \\
\hline performance & 17 & $\mathrm{TiO}_{2}$ & 11 \\
\hline stability & 17 & catalytic-activity & 10 \\
\hline particles & 16 & hydrogen-peroxide & 10 \\
\hline water & 16 & mechanical-properties & 10 \\
\hline conversion & 15 & & \\
\hline
\end{tabular}

Fig. 2 presents the conceptual structure map of the proposed study. According to our survey, green chemistry, water, heterogeneous catalysis and aqueous-solution build a structure on mesoporous 
materials. Moreover, conversion, guest molecules, triblock, oxidation, heterogeneous catalysts set the corner of other structure of the study.

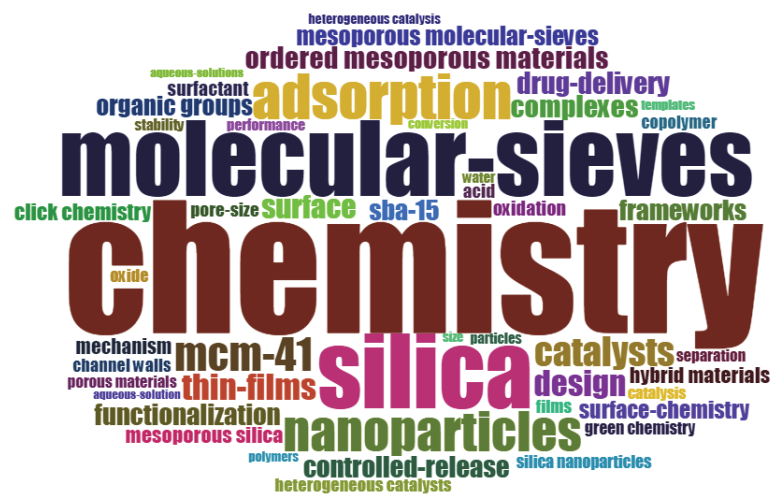

Fig. 1. Demographic of the trend of different words used in literature

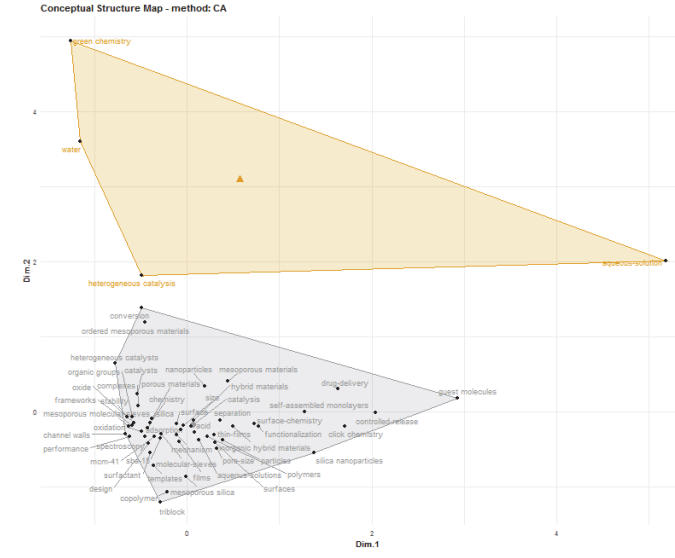

Fig. 2. Factorial analysis

\subsection{Country Scientific Production}

According to Fig. 3, the distribution of scientific production by various countries and as we can observe, the largest scientific productions are associated with United States, China and Australia.

\section{Country Scientific Production}

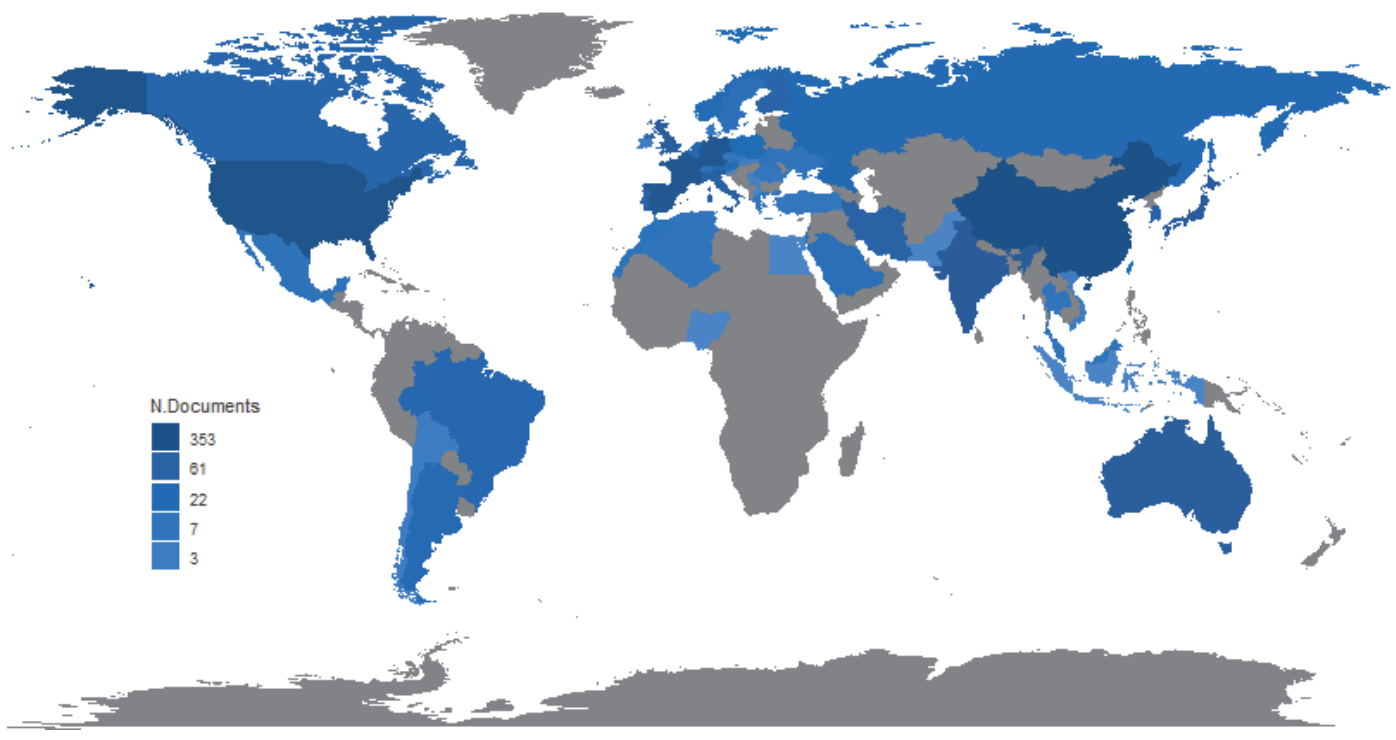

Fig. 3. Country Scientific Production

\subsection{Corresponding author's country}

Our survey demonstrates that researchers from the China have maintained the most contribution in this field followed by the researchers from the United States and Germany. Fig. 4 shows the details of our survey. Moreover, we see a good collaboration between most countries with other countries. 


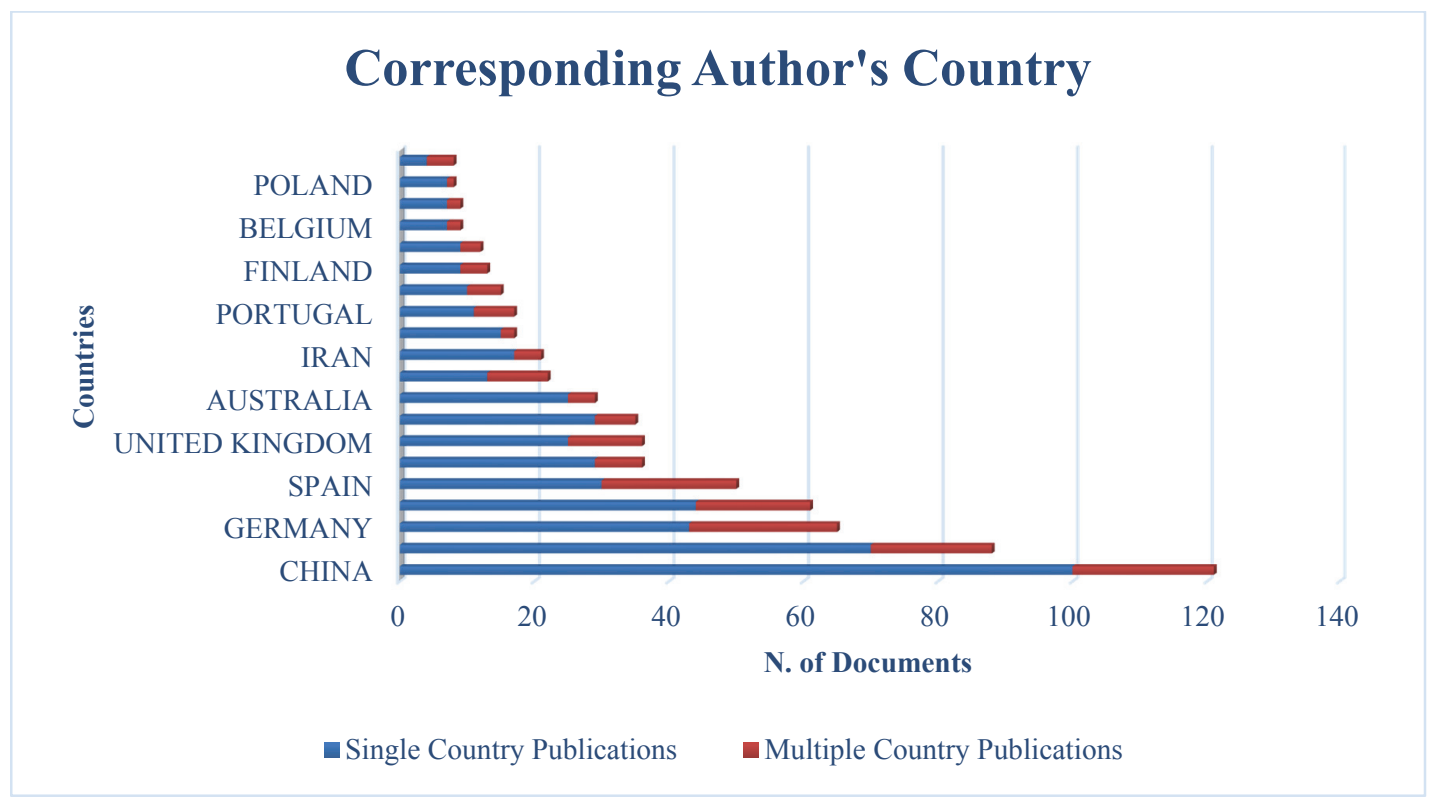

Fig. 4 Corresponding author's country

\subsection{The frequency distribution of sources}

In this research, most articles from the sources shown in Fig. 5 are from the Journal of ChemistryA European Journal with 64 articles, followed by Angewandte Chemie-International Edition with 47 articles.

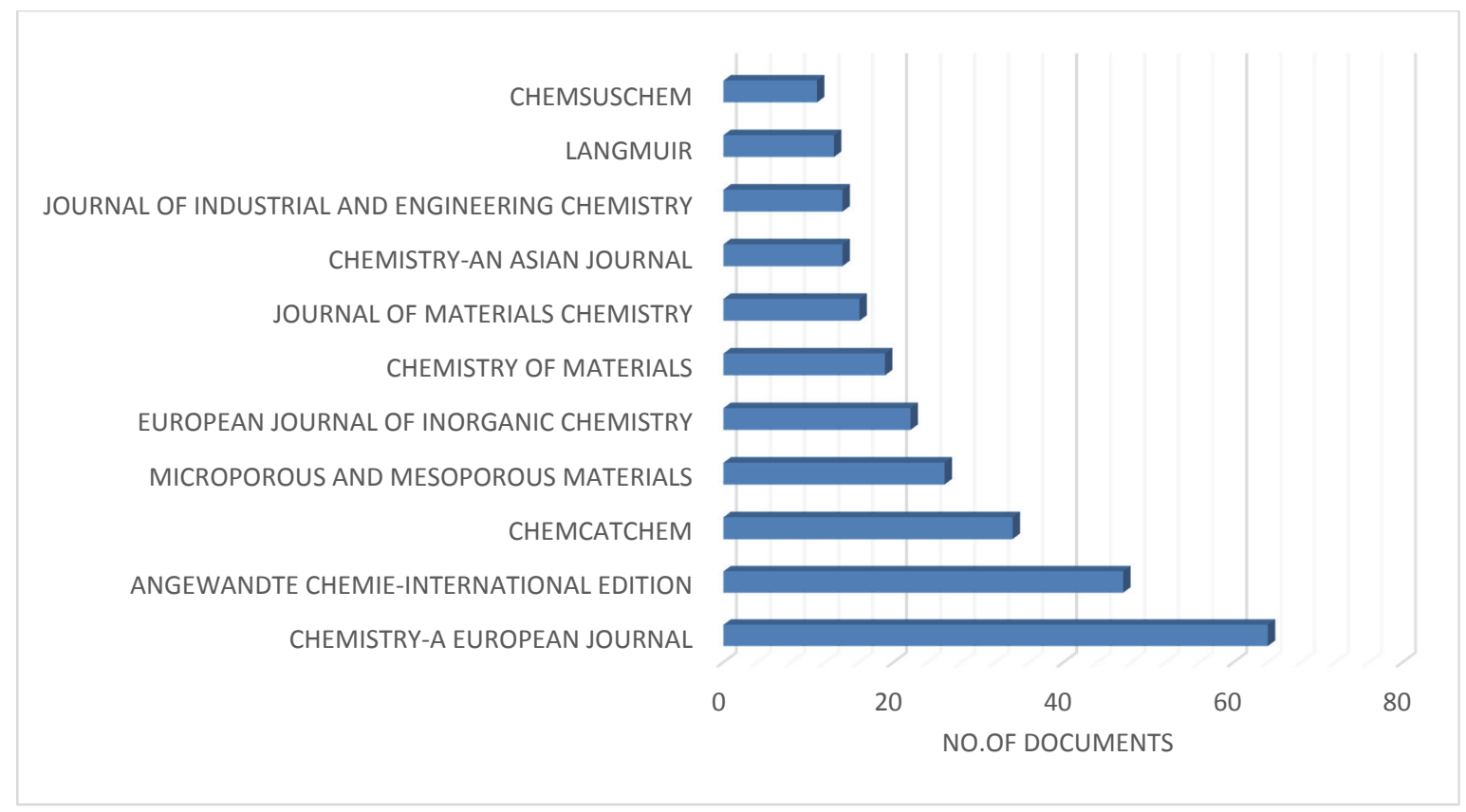

Fig. 5. Most Relevant Source

\subsection{Collaboration network and Cluster classification}

Fig. 6 shows the Author's Collaboration Network. As we can observe from the results of Table 2 and Fig. 7, there are three clusters associated with mesoporous materials in chemistry. In the first cluster, silica appears to be the most important word followed by molecular-sieves and MCM-41. Organic- 
group is the most important word in the second cluster followed by hybrid materials. In cluster 3 , nanoparticles appears to be the most important word followed by functionalization.

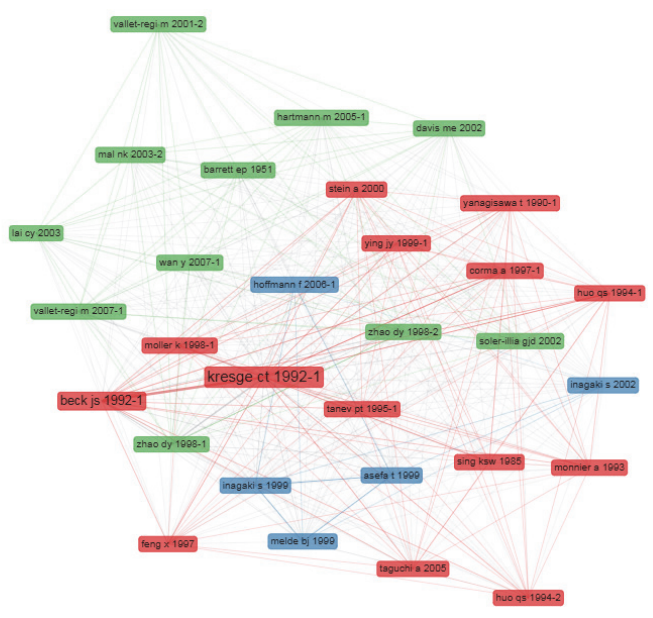

Fig. 6. Author's Collaboration Network

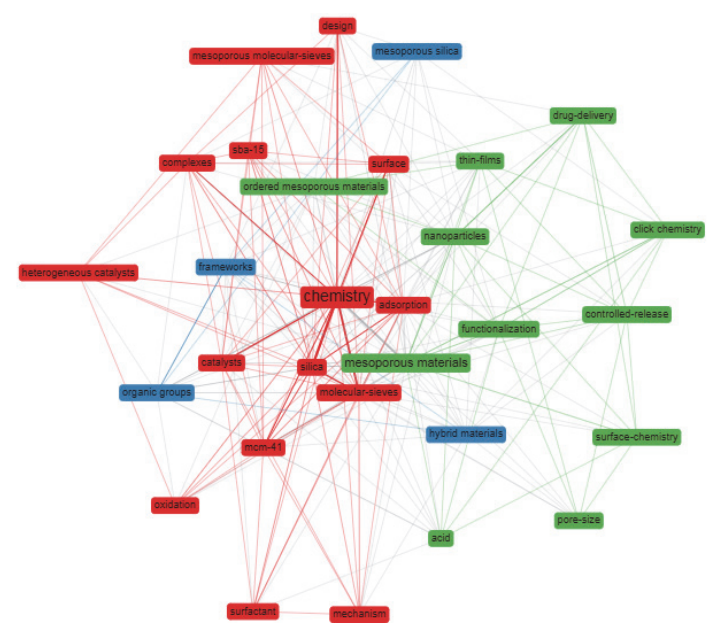

Fig. 7. Demographic of three clusters

\section{Table 2}

Demographic of three clusters

\begin{tabular}{|c|c|c|c|}
\hline Term & Cluster & Term & Cluster \\
\hline molecular-sieves & 1 & organic groups & 2 \\
\hline silica & 1 & mesoporous silica & 2 \\
\hline mechanism & 1 & frameworks & 2 \\
\hline chemistry & 1 & hybrid materials & 2 \\
\hline catalysts & 1 & thin-films & 3 \\
\hline heterogeneous catalysts & 1 & mesoporous materials & 3 \\
\hline complexes & 1 & pore-size & 3 \\
\hline surface & 1 & functionalization & 3 \\
\hline MCM-41 & 1 & controlled-release & 3 \\
\hline surfactant & 1 & click chemistry & 3 \\
\hline adsorption & 1 & drug-delivery & 3 \\
\hline Design & 1 & ordered mesoporous materials & 3 \\
\hline mesoporous molecular-sieves & 1 & nanoparticles & 3 \\
\hline SBA-15 & 1 & acid & 3 \\
\hline oxidation & 1 & surface-chemistry & 3 \\
\hline
\end{tabular}

In terms of the average citation, papers published by researchers in Canada, Australia, and France have received the highest citations. Fig. 8 shows the results of the collaborations among various countries. As we can observe from the results of Fig. 8, there was a strong collaboration from the researchers in the United States from one side and other countries which was the highest to Canada, Australia and China, respectively. 


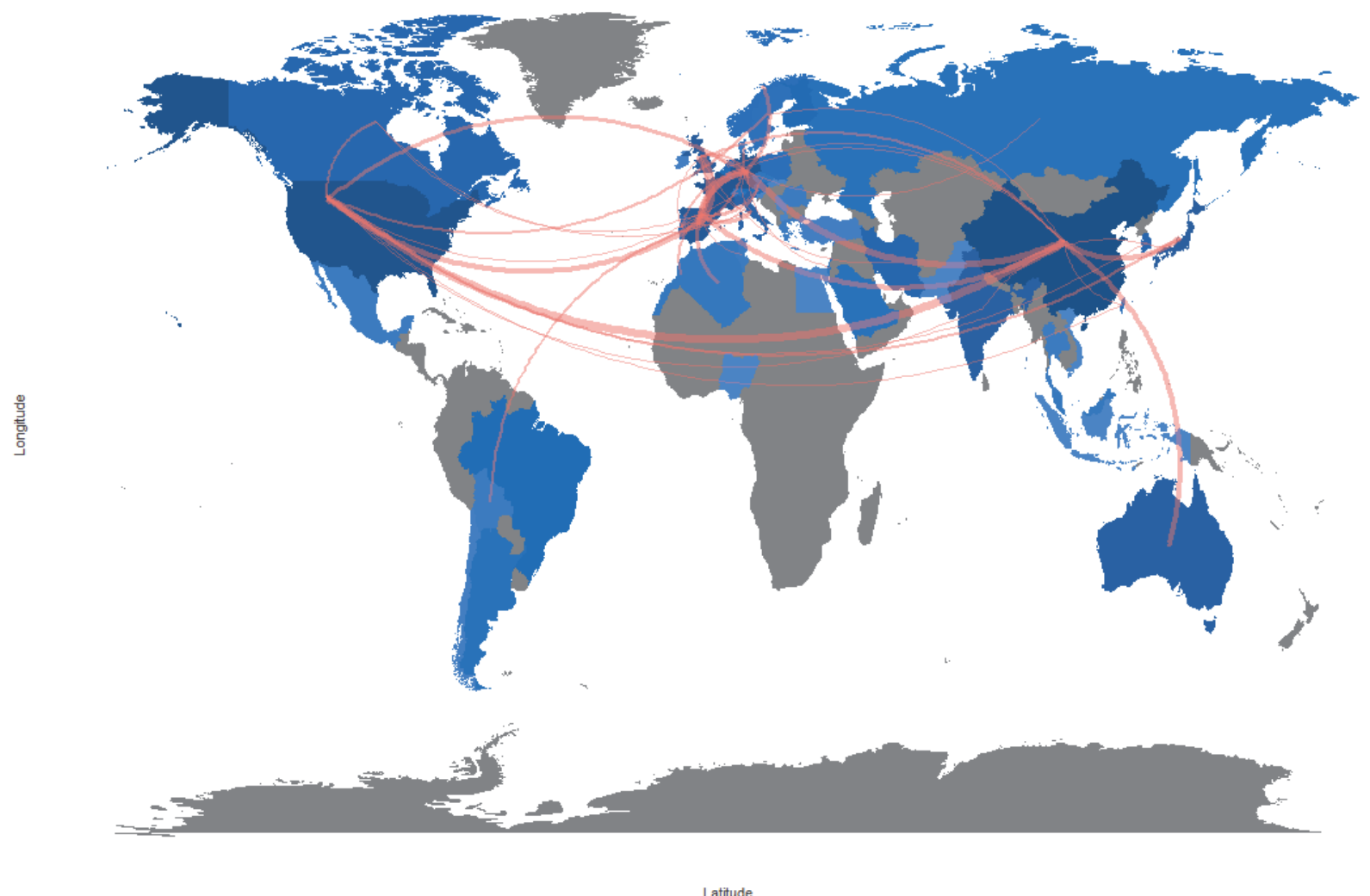

Fig. 8. Country Collaboration map

Fig. 9 demonstrates how many articles have been written by the authors with the highest number of articles during the time, and how many citations each one received. The size of each circle indicates the number of articles and the amount of boldness of the circles indicates the number of citations in that year.

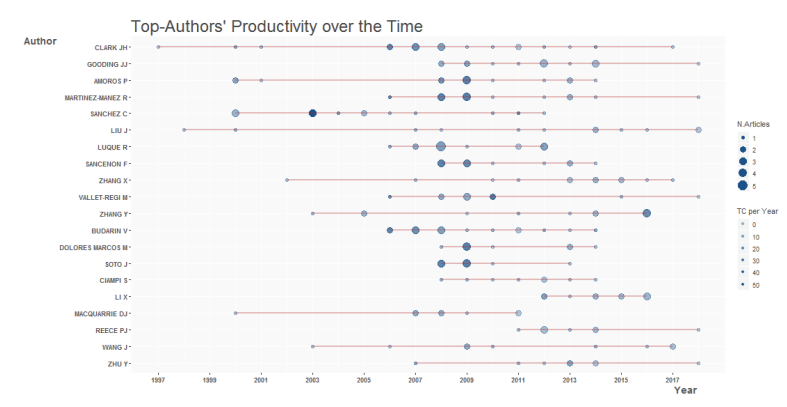

Fig. 9. Top-Authors' productivity over the time

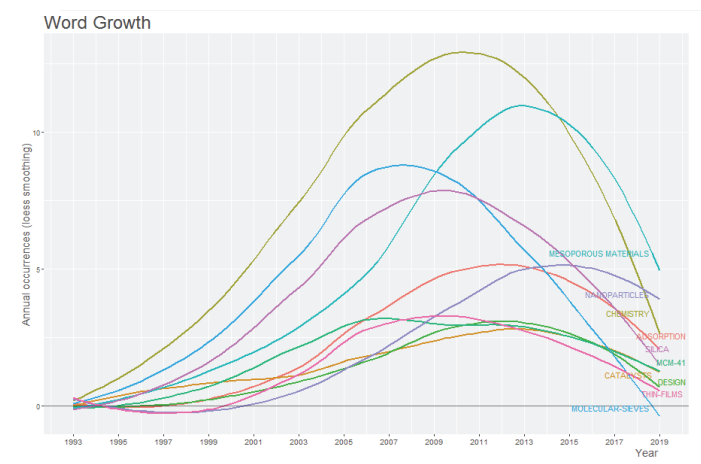

Fig. 10. The trend on Word growth

\subsection{Thematic map}

Co-word analysis draws clusters of keywords considered as themes. In the strategic diagram presented in Fig. 11 the vertical axis measures the density - i.e., the strength of the internal links within a cluster represented by a theme -, and the horizontal vertical axis the centrality - i.e. the strength of the links between the theme and other themes in the map. ${ }^{203-209}$ 
Thematic map is a very intuitive plot and we can analyze themes according to the quadrant in which they are placed:

(Q1) upper-right quadrant: motor-themes;

(Q2) lower-right quadrant: basic themes;

(Q3) lower-left quadrant: emerging or disappearing themes;

(Q4) upper-left quadrant: very specialized/ niche themes.

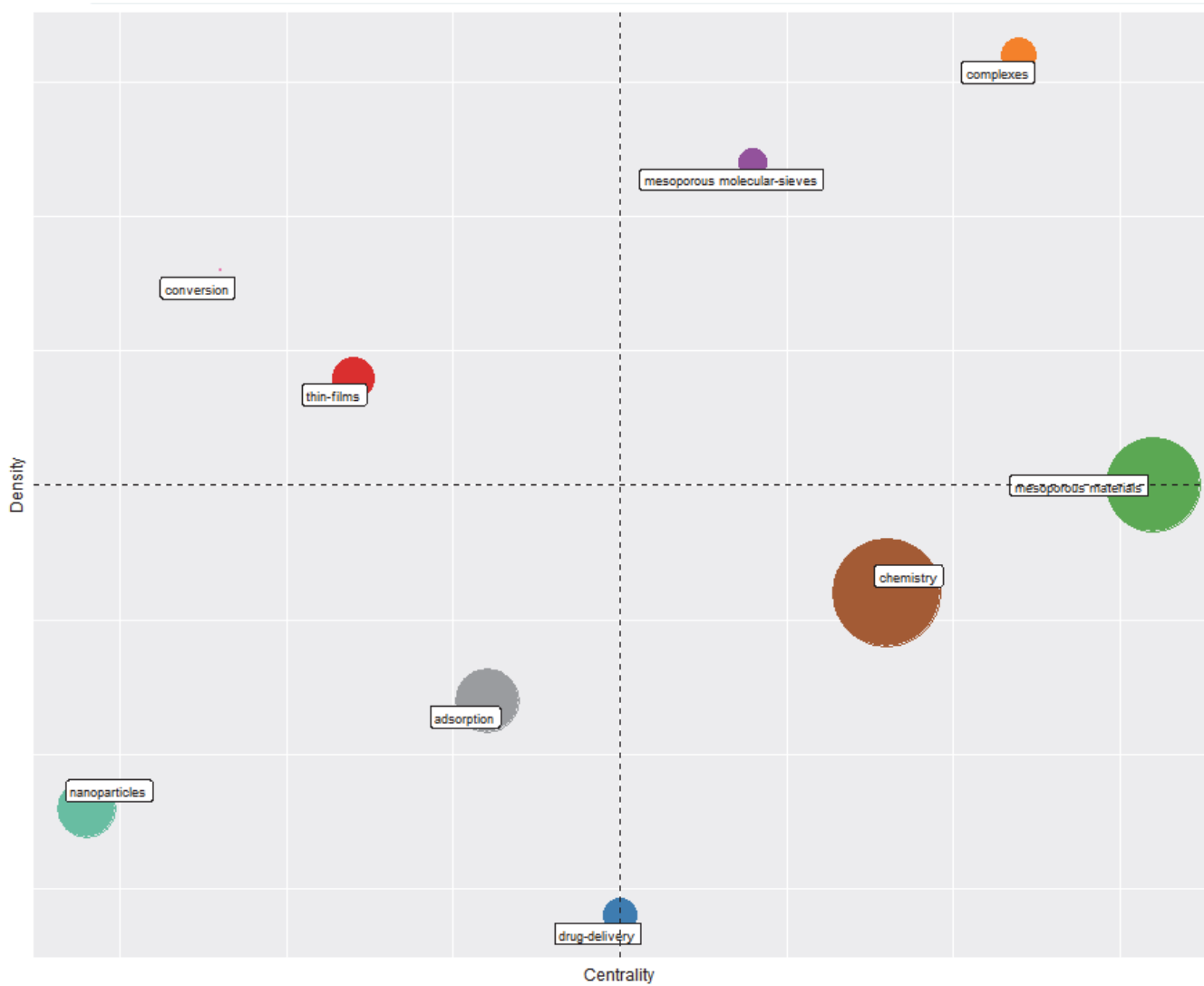

Fig. 11. Thematic Map

Hence, the themes with the highest internal coherence and closest relationship to other themes appear in the first quadrant (the upper right part of the graph) and this includes complexes and mesoporous molecular-sieves and these two keywords are considered as motor keywords. In the second quarter, chemistry as a general word plays the basic role for scientific development. Themes in this quadrant are important for a research field but are not developed and they are considered as emerging areas of research. This group includes Absorption, Nanoparticles and Drug-delivery. This quadrant includes transversal and general, basic themes, which include conversion and thin-films.

\subsection{Intellectual Structure, Historiographic}

The historiographic map is a graph proposed by E. Garfield to represent a chronological network map of the most relevant direct citations resulting from a bibliographic collection. The citation network technique does provide the scholar with a new modus operandi which may significantly affect future historiography. The results of citation cooperation is given in Fig. 12. Moreover, there are some good 
collaborations network between various groups of authors demonstrated in Fig. 13. The first group consists of the group with $\mathrm{Li}^{71}$, Liu et al. ${ }^{63,83,154,173}$, Zhang et al. ${ }^{46,72,82,93,104,112,126,143,182}$, Tan et al. ${ }^{133,160}$, and Gao et al. ${ }^{177}$. The second group include 9 researchers including Marcos et al. 42,51,84,85,86,153, Sancenon et al. ${ }^{10,153}$, Martinez et al. ${ }^{202}$, Bernardos et al. ${ }^{51,119}$, Soto et al. ${ }^{42,85,119,153}$, Amoros et al. ${ }^{43}$, Marcos et al. ${ }^{42,51,84,85,86,119,153}$, Aznar et al. ${ }^{51,119,153}$ and Coll et al. ${ }^{84,119,153,176}$. The third group is associated with five authors including Budarin et al. ${ }^{26,57,120,149,157,166}$ Macquarrie et al. ${ }^{95,120,155}$, Clark et al. ${ }^{26,27,57,95,120,124,149,155,166}$, Luque et al. ${ }^{52,120149,155}$ and Budarin et al. ${ }^{26,52,57,95,124,149,155,157}$. The fourth group is the cooperation among four researchers including Boissiere et al. ${ }^{31}$, Grosso et al., $6,24,31,48,108,179$, Sanchez et al. ${ }^{6,24,48,79,107,113,162,179,180}$ and Antonietti et al. ${ }^{4,71,108,161}$. The other group of researchers include Gooding et al. ${ }^{73,121,184}$, Ciampi et al. ${ }^{121,169}$, Zhu et al. ${ }^{59,89,97,110,129,173,177,181}$ and Reece et al. ${ }^{108}$. The other collaboration was executed among Biossiere, Sanchez et al. $6,24,31,48,79,107,113,162,179,180$, Grosso 6, 24,31, 48, 108, 179 and Antonierri et al. ${ }^{4,71,108,161}$.

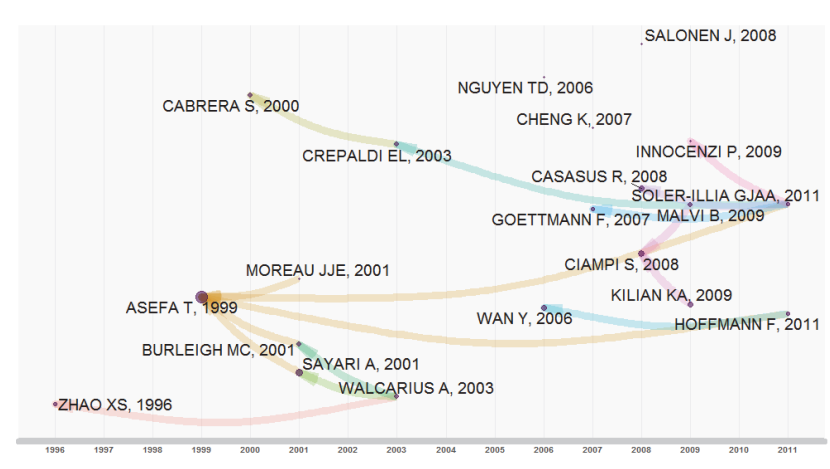

Fig. 12. Historical direct citation network
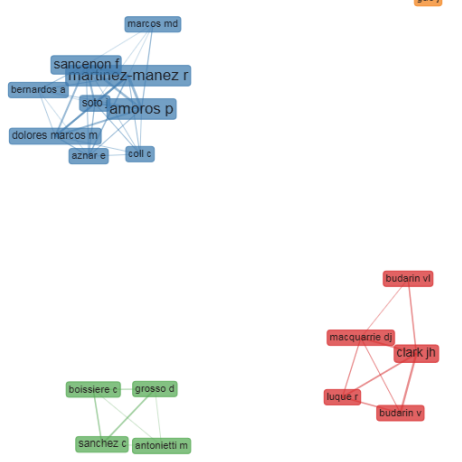

Fig. 13. World collaboration network

\section{Conclusion}

This study has tried to provide a comprehensive view of the scientific papers between 1900 to the first month of 2019 on mesoporous materials in chemistry. This research has shown researchers from China, Germany, Spain, United Kingdom and Australia have contributed the most in this area. Moreover, green chemistry, water, heterogenous catalysis and aqueous-solution have built a structure on mesoporous materials. In addition, the themes with the highest internal coherence and closest relationship to other themes have included complexes and mesoporous molecular-sieves and these keywords were considered as motor keywords. Absorption, Nanoparticles and Drug-delivery were detected as the emerging keywords and future studies could be concentrated on these issues.

\section{References}

1. Rouquerol, J., Avnir, D., Fairbridge, C. W., Everett, D. H., Haynes, J. M., Pernicone, N., ... \& Unger, K. K. (1994). Recommendations for the characterization of porous solids (Technical Report). Pure Appl. Chem., 66(8), 1739-1758.

2. Eftekhari, A., \& Fan, Z. (2017). Ordered mesoporous carbon and its applications for electrochemical energy storage and conversion. Mat. Chem. Front., 1(6), 1001-1027.

3. Asefa, T., MacLachlan, M. J., Coombs, N., \& Ozin, G. A. (1999). Periodic mesoporous organosilicas with organic groups inside the channel walls. Nature, 402(6764), 867.

4. Wang, Y., Wang, X., \& Antonietti, M. (2012). Polymeric graphitic carbon nitride as a heterogeneous organocatalyst: from photochemistry to multipurpose catalysis to sustainable chemistry. Angew. Chem. Int. Edit., 51(1), 68-89. 
5. Rolison, D. R. (2003). Catalytic nanoarchitectures--the importance of nothing and the unimportance of periodicity. Science, 299(5613), 1698-1701.

6. Crepaldi, E. L., Soler-Illia, G. J. D. A., Grosso, D., Cagnol, F., Ribot, F., \& Sanchez, C. (2003). Controlled formation of highly organized mesoporous titania thin films: from mesostructured hybrids to mesoporous nanoanatase TiO2. J. Am. Chem. Soc., 125(32), 9770-9786.

7. Sayari, A., \& Hamoudi, S. (2001). Periodic mesoporous silica-based organic- inorganic nanocomposite materials. Chem. Matl., 13(10), 3151-3168.

8. Zhao, X. S., Lu, G. Q., \& Millar, G. J. (1996). Advances in mesoporous molecular sieve MCM-41. Ind. Eng. Chem. Res., 35(7), 2075-2090.

9. Yan, X., Yu, C., Zhou, X., Tang, J., \& Zhao, D. (2004). Highly ordered mesoporous bioactive glasses with superior in vitro bone-forming bioactivities. Angew. Chem. Int. Edit., 43(44), 5980-5984.

10. Descalzo, A. B., Martínez-Máñez, R., Sancenon, F., Hoffmann, K., \& Rurack, K. (2006). The supramolecular chemistry of organic-inorganic hybrid materials. Angew. Chem. Int. Edit., 45(36), 5924-5948.

11. Indrakanti, V. P., Kubicki, J. D., \& Schobert, H. H. (2009). Photoinduced activation of CO 2 on Tibased heterogeneous catalysts: Current state, chemical physics-based insights and outlook. Energy \& Environmental Science, 2(7), 745-758.

12. Vallet-Regí, M. (2006). Ordered mesoporous materials in the context of drug delivery systems and bone tissue engineering. Chem.-Eur. J., 12(23), 5934-5943.

13. Angelos, S., Yang, Y. W., Patel, K., Stoddart, J. F., \& Zink, J. I. (2008). pH-responsive supramolecular nanovalves based on cucurbit [6] uril pseudorotaxanes. Angew. Chem., 120(12), 2254-2258.

14. Park, C., Oh, K., Lee, S. C., \& Kim, C. (2007). Controlled release of guest molecules from mesoporous silica particles based on a pH-responsive polypseudorotaxane motif. Angew. Chem. Int. Edit., 46(9), 1455-1457.

15. Landers, J., Gor, G. Y., \& Neimark, A. V. (2013). Density functional theory methods for characterization of porous materials. Colloids and Surfaces A-Physicochemical and Engineering Aspects, 437, 3-32.

16. Lin, H. P., \& Mou, C. Y. (2002). Structural and morphological control of cationic surfactant-templated mesoporous silica. Accounts Chem. Res., 35(11), 927-935.

17. Salonen, J., Kaukonen, A. M., Hirvonen, J., \& Lehto, V. P. (2008). Mesoporous silicon in drug delivery applications. J. of pharm. Sci., 97(2), 632-653.

18. Chal, R., Gerardin, C., Bulut, M., \& Van Donk, S. (2011). Overview and industrial assessment of synthesis strategies towards zeolites with mesopores. ChemCatChem, 3(1), 67-81.

19. Arends, I. W., Sheldon, R. A., Wallau, M., \& Schuchardt, U. (1997). Oxidative transformations of organic compounds mediated by redox molecular sieves. Angew. Chemie Int. Edit., 36(11), 1144-1163.

20. Arcos, D., \& Vallet-Regí, M. (2010). Sol-gel silica-based biomaterials and bone tissue regeneration. Acta Biomater, 6(8), 2874-2888.

21. Wan, Y., Yang, H., \& Zhao, D. (2006). "Host- Guest" chemistry in the synthesis of ordered nonsiliceous mesoporous materials. Accounts Chem. Res., 39(7), 423-432.

22. Yokoi, T., Yoshitake, H., \& Tatsumi, T. (2004). Synthesis of amino-functionalized MCM-41 via direct co-condensation and post-synthesis grafting methods using mono-, di-and tri-aminoorganoalkoxysilanes. J. of Mater. Chem., 14(6), 951-957.

23. Lee, C. H., Lin, T. S., \& Mou, C. Y. (2009). Mesoporous materials for encapsulating enzymes. Nano today, 4(2), 165-179.

24. Grosso, D., Boissière, C., Smarsly, B., Brezesinski, T., Pinna, N., Albouy, P. A., ... \& Sanchez, C. (2004). Periodically ordered nanoscale islands and mesoporous films composed of nanocrystalline multimetallic oxides. Nat. Mater., 3(11), 787.

25. Papaefstathiou, G. S., \& MacGillivray, L. R. (2003). Inverted metal-organic frameworks: solid-state hosts with modular functionality. Coordin. Chem. Rev., 246(1-2), 169-184.

26. Karimi, B., Abedi, S., Clark, J. H., \& Budarin, V. (2006). Highly Efficient Aerobic Oxidation of Alcohols Using a Recoverable Catalyst: The Role of Mesoporous Channels of SBA-15 in Stabilizing Palladium Nanoparticles. Angew. Chem. Int. Edit., 45(29), 4776-4779.

27. Wilson, K., \& Clark, J. H. (2000). Solid acids and their use as environmentally friendly catalysts in organic synthesis. Pure Appl. Chem., 72(7), 1313-1319. 
28. Walcarius, A., Etienne, M., \& Lebeau, B. (2003). Rate of access to the binding sites in organically modified silicates. 2. Ordered mesoporous silicas grafted with amine or thiol groups. Chem. Mater., 15(11), 2161-2173.

29. Clearfield, A. (1998). Organically pillared micro-and mesoporous materials. Chem. of Mater., 10(10), 2801-2810.

30. Chen, C., Geng, J., Pu, F., Yang, X., Ren, J., \& Qu, X. (2011). Polyvalent Nucleic Acid/Mesoporous Silica Nanoparticle Conjugates: Dual Stimuli-Responsive Vehicles for Intracellular Drug Delivery. Angew. Chem. Int. Ent., 50(4), 882-886.

31. Boissiere, C., Grosso, D., Chaumonnot, A., Nicole, L., \& Sanchez, C. (2011). Aerosol route to functional nanostructured inorganic and hybrid porous materials. Adv. Mater., 23(5), 599-623.

32. Moreau, J. J., Vellutini, L., Chi Man, M. W., Bied, C., Bantignies, J. L., Dieudonné, P., \& Sauvajol, J. L. (2001). Self-organized hybrid silica with long-range ordered lamellar structure. J. Am. Chem. Soc., 123(32), 7957-7958.

33. Weitkamp, J., Hunger, M., \& Rymsa, U. (2001). Base catalysis on Micropor. Mesopor. Mat.: recent progress and perspectives. Micropor. Mesopor. Mat., 48(1-3), 255-270.

34. Nguyen, T. D., Leung, K. C. F., Liong, M., Pentecost, C. D., Stoddart, J. F., \& Zink, J. I. (2006). Construction of a pH-driven supramolecular nanovalve. Org. Lett., 8(15), 3363-3366.

35. Thomas, J. M., \& Raja, R. (2008). Exploiting nanospace for asymmetric catalysis: confinement of immobilized, single-site chiral catalysts enhances enantioselectivity. Accounts Chem. Res., 41(6), 708720.

36. Zhao, D., Peidong, Y., \& Qisheng, H. (1998). Topological construction of mesoporous materials. Curr. Opin. Solid St. M. 3(1), 111-121.

37. Hartmann, M., Kullmann, S., \& Keller, H. (2010). Wastewater treatment with heterogeneous Fentontype catalysts based on porous materials. J. Mater. Chem., 20(41), 9002-9017.

38. Khushalani, D., Kuperman, A., Ozin, G. A., Tanaka, K., Coombs, N., Olken, M. M., \& Garcés, J. (1995). Metamorphic materials: restructuring siliceous mesoporous materials. Adv. Mater., 7(10), 842846.

39. Hoffmann, F., \& Fröba, M. (2011). Vitalising porous inorganic silica networks with organic functions-PMOs and related hybrid materials. Chem. Soc. Rev., 40(2), 608-620.

40. Wang, D., Xie, T., Peng, Q., \& Li, Y. (2008). Ag, Ag2S, and Ag2Se nanocrystals: synthesis, assembly, and construction of mesoporous structures. J. Am. Chem. Soc., 130(12), 4016-4022.

41. Yu, J., Cui, Y., Wu, C., Yang, Y., Wang, Z., O'Keeffe, M., ... \& Qian, G. (2012). Second-order nonlinear optical activity induced by ordered dipolar chromophores confined in the pores of an anionic metal-organic framework. Angew. Chem-Ger Edit, 124(42), 10694-10697.

42. Casasús, R., Climent, E., Marcos, M. D., Martínez-Máñez, R., Sancenón, F., Soto, J., ... \& Ruiz, E. (2008). Dual aperture control on $\mathrm{pH}$-and anion-driven supramolecular nanoscopic hybrid gate-like ensembles. J. Am. Chem. Soc., 130(6), 1903-1917.

43. Cabrera, S., El Haskouri, J., Guillem, C., Latorre, J., Beltrán-Porter, A., Beltrán-Porter, D., ... \& Amoros, P. (2000). Generalised syntheses of ordered mesoporous oxides: the atrane route. Solid State Sci., 2(4), 405-420.

44. Jérôme, F., Pouilloux, Y., \& Barrault, J. (2008). Rational design of solid catalysts for the selective use of glycerol as a natural organic building block. Chem. Sus. Chem., 1(7), 586-613.

45. Cundy, C. S. (1998). Microwave techniques in the synthesis and modification of zeolite catalysts. A review. Collect. Czech. Chem. C., 63(11), 1699-1723.

46. Qiu, L. G., Xu, T., Li, Z. Q., Wang, W., Wu, Y., Jiang, X., ... \& Zhang, L. D. (2008). Hierarchically Micro-and Mesoporous Metal-Organic Frameworks with Tunable Porosity. Angew. Chem-Ger Edit, 120(49), 9629-9633.

47. Ariga, K., Vinu, A., Hill, J. P., \& Mori, T. (2007). Coordination chemistry and supramolecular chemistry in mesoporous nanospace. Coordination Chemistry Reviews, 251(21-24), 2562-2591.

48. Sanchez, C., Galo, J., Ribot, F., \& Grosso, D. (2003). Design of functional nano-structured materials through the use of controlled hybrid organic-inorganic interfaces. Comptes Rendus Chimie, 6(8-10), 1131-1151.

49. Soler-Illia, G. D. A., \& Innocenzi, P. (2006). Mesoporous hybrid thin films: The physics and chemistry beneath. Chem.-A Europ. J., 12(17), 4478-4494. 
50. Braunstein, P. (2004). Functional ligands and complexes for new structures, homogeneous catalysts and nanomaterials. J. Organom. Chgem., 689(24), 3953-3967.

51. Bernardos, A., Aznar, E., Marcos, M. D., Martínez-Máñez, R., Sancenón, F., Soto, J., ... \& Amorós, P. (2009). Enzyme-responsive controlled release using mesoporous silica supports capped with lactose. Angew. Chem-Ger Edit, 121(32), 5998-6001.

52. Budarin, V., Clark, J. H., Hardy, J. J., Luque, R., Milkowski, K., Tavener, S. J., \& Wilson, A. J. (2006). Starbons: New starch-derived mesoporous carbonaceous materials with tunable properties. Angew. Chem-Ger Edit, 118(23), 3866-3870.

53. Soler-Illia, G. J., \& Azzaroni, O. (2011). Multifunctional hybrids by combining ordered mesoporous materials and macromolecular building blocks. Chem. Soc. Rev., 40(2), 1107-1150.

54. Fang, Q. R., Yuan, D. Q., Sculley, J., Li, J. R., Han, Z. B., \& Zhou, H. C. (2010). Functional mesoporous metal- organic frameworks for the capture of heavy metal ions and size-selective catalysis. Inorg. Chem., 49(24), 11637-11642.

55. Ozin, G. A., Hou, K., Lotsch, B. V., Cademartiri, L., Puzzo, D. P., Scotognella, F., ... \& Thomson, J. (2009). Nanofabrication by self-assembly. Mater. Today, 12(5), 12-23.

56. Han, Y., Li, D., Zhao, L., Song, J., Yang, X., Li, N., ... \& Meng, X. (2003). High-Temperature Generalized Synthesis of Stable Ordered Mesoporous Silica-Based Materials by Using FluorocarbonHydrocarbon Surfactant Mixtures. Angew. Chem-Ger Edit, 115(31), 3761-3765.

57. Karimi, B., Biglari, A., Clark, J. H., \& Budarin, V. (2007). Green, Transition-Metal-Free Aerobic Oxidation of Alcohols Using a Highly Durable Supported Organocatalyst. Angew. Chem. Int. Ent., 46(38), 7210-7213.

58. Botterhuis, N. E., Sun, Q., Magusin, P. C., Van Santen, R. A., \& Sommerdijk, N. A. (2006). Hollow silica spheres with an ordered pore structure and their application in controlled release studies. Chem.A Europ. J., 12(5), 1448-1456.

59. Zhu, Y., \& Fujiwara, M. (2007). Installing dynamic molecular photomechanics in mesopores: a multifunctional controlled-release nanosystem. Angew. Chem. Int. Ent., 46(13), 2241-2244.

60. Burleigh, M. C., Markowitz, M. A., Spector, M. S., \& Gaber, B. P. (2001). Amine-functionalized periodic mesoporous organosilicas. Chem. Mater., 13(12), 4760-4766.

61. Holland, B. T., Isbester, P. K., Blanford, C. F., Munson, E. J., \& Stein, A. (1997). Synthesis of ordered aluminophosphate and galloaluminophosphate mesoporous materials with anion-exchange properties utilizing polyoxometalate cluster/surfactant salts as precursors. J. Am. Chem. Soc., 119(29), 67966803.

62. Spitler, E. L., Colson, J. W., Uribe-Romo, F. J., Woll, A. R., Giovino, M. R., Saldivar, A., \& Dichtel, W. R. (2012). Lattice expansion of highly oriented 2D phthalocyanine covalent organic framework films. Angew. Chem-Ger Edit, 124(11), 2677-2681.

63. Liu, J., Shin, Y., Nie, Z., Chang, J. H., Wang, L. Q., Fryxell, G. E., ... \& Exarhos, G. J. (2000). Molecular assembly in ordered mesoporosity: A new class of highly functional nanoscale materials. The J. of Physical Chem. A, 104(36), 8328-8339.

64. Fischbach, A., Klimpel, M. G., Widenmeyer, M., Herdtweck, E., Scherer, W., \& Anwander, R. (2004). Stereospecific Polymerization of Isoprene with Molecular and MCM-48-Grafted Lanthanide (III) Tetraalkylaluminates. Angew. Chem-Ger Edit, 116(17), 2284-2289.

65. Behrens, P. (1993). Mesoporous inorganic solids. Adv. Mater., 5(2), 127-132.

66. MacLachlan, M. J., Asefa, T., \& Ozin, G. A. (2000). Writing on the wall with a new synthetic quill. Chem.-A Europ.J., 6(14), 2507-2511.

67. Erlebacher, J., \& Seshadri, R. (2009). Hard materials with tunable porosity. Mrs Bulletin, 34(8), 561568.

68. Ghanbari-Siahkali, A., Philippou, A., Dwyer, J., \& Anderson, M. W. (2000). The acidity and catalytic activity of heteropoly acid on MCM-41 investigated by MAS NMR, FTIR and catalytic tests. Appl. Catal. B-Gen., 192(1), 57-69.

69. Kelly, J. A., Giese, M., Shopsowitz, K. E., Hamad, W. Y., \& MacLachlan, M. J. (2014). The development of chiral nematic mesoporous materials. Accounts Chem. Res., 47(4), 1088-1096.

70. Zou, R., Abdel-Fattah, A. I., Xu, H., Zhao, Y., \& Hickmott, D. D. (2010). Storage and separation applications of nanoporous metal-organic frameworks. CrystEngComm, 12(5), 1337-1353. 
71. Li, X. H., Wang, X., \& Antonietti, M. (2012). Solvent-free and metal-free oxidation of toluene using $\mathrm{O} 2$ and g-C3N4 with nanopores: nanostructure boosts the catalytic selectivity. Acs Catalysis, 2(10), 2082-2086.

72. Huang, Y., Cai, H., Yu, T., Zhang, F., Zhang, F., Meng, Y., ... \& Zhao, D. (2007). Formation of Mesoporous Carbon With a Face-Centered-Cubic Fd 3 m Structure and Bimodal Architectural Pores From the Reverse Amphiphilic Triblock Copolymer PPO-PEO-PPO. Angew. Chem-Ger Edit, 119(7), 1107-1111.

73. Kilian, K. A., Böcking, T., \& Gooding, J. J. (2009). The importance of surface chemistry in mesoporous materials: lessons from porous silicon biosensors. Chem. Commun., (6), 630-640.

74. Kanatzidis, M. G. (2007). Beyond silica: Nonoxidic mesostructured materials. Adv. Mater., 19(9), 1165-1181.

75. Kandambeth, S., Shinde, D. B., Panda, M. K., Lukose, B., Heine, T., \& Banerjee, R. (2013). Enhancement of Chemical Stability and Crystallinity in Porphyrin-Containing Covalent Organic Frameworks by Intramolecular Hydrogen Bonds. Angew. Chem. Int. Ent., 52(49), 13052-13056.

76. Chen, S., Duan, J., Tang, Y., \& Zhang Qiao, S. (2013). Hybrid hydrogels of porous graphene and nickel hydroxide as advanced supercapacitor materials. Chemistry-A European Journal, 19(22), 7118-7124.

77. Xu, W., Riikonen, J., \& Lehto, V. P. (2013). Mesoporous systems for poorly soluble drugs. Int. J. Pharm., 453(1), 181-197.

78. Qian, K. K., \& Bogner, R. H. (2012). Application of mesoporous silicon dioxide and silicate in oral amorphous drug delivery systems. J Pharm. Sci., 101(2), 444-463.

79. Goettmann, F., \& Sanchez, C. (2007). How does confinement affect the catalytic activity of mesoporous materials?. J. Mater. Chem., 17(1), 24-30.

80. Kresge, C. T., \& Roth, W. J. (2013). The discovery of mesoporous molecular sieves from the twenty year perspective. Chem. Soc. Rev., 42(9), 3663-3670.

81. Vallet-Regí, M., Balas, F., Colilla, M., \& Manzano, M. (2008). Bone-regenerative bioceramic implants with drug and protein controlled delivery capability. Prog. Solid State. Ch., 36(3), 163-191.

82. Zeng, W., Qian, X. F., Zhang, Y. B., Yin, J., \& Zhu, Z. K. (2005). Organic modified mesoporous MCM-41 through solvothermal process as drug delivery system. Mater. Res. Bull., 40(5), 766-772.

83. Feng, D., Wang, K., Su, J., Liu, T. F., Park, J., Wei, Z., ... \& Zhou, H. C. (2015). A highly stable zeotype mesoporous zirconium metal-organic framework with ultralarge pores. Angew. Chem. Int. Ent., 54(1), 149-154.

84. Ros-Lis, J. V., Casasús, R., Comes, M., Coll, C., Marcos, M. D., Martínez-Máñez, R., ... \& Garró, N. (2008). A mesoporous 3D hybrid material with dual functionality for $\mathrm{Hg} 2+$ detection and adsorption. Chem.-A Europ. J., 14(27), 8267-8278.

85. Climent, E., Marcos, M. D., Martínez-Máñez, R., Sancenón, F., Soto, J., Rurack, K., \& Amorós, P. (2009). The determination of methylmercury in real samples using organically capped mesoporous inorganic materials capable of signal amplification. Angew. Chem-Ger Edit, 121(45), 8671-8674.

86. Cabrera, S., El Haskouri, J., Beltrán-Porter, A., Beltrán-Porter, D., Marcos, M. D., \& Amorós, P. (2000). Enhanced surface area in thermally stable pure mesoporous TiO2. Solid State Sci., 2(5), 513 518.

87. Wang, J., Johnston-Peck, A. C., \& Tracy, J. B. (2009). Nickel phosphide nanoparticles with hollow, solid, and amorphous structures. Chem. Mater., 21(19), 4462-4467.

88. Salonen, J., \& Lehto, V. P. (2008). Fabrication and chemical surface modification of mesoporous silicon for biomedical applications. Chem. Eng. J., 137(1), 162-172.

89. Polarz, S., Orlov, A. V., Schüth, F., \& Lu, A. H. (2007). Preparation of High-Surface-Area Zinc Oxide with Ordered Porosity, Different Pore Sizes, and Nanocrystalline Walls. Chem.-A Europ. J., 13(2), 592-597.

90. Zareyee, D., \& Karimi, B. (2007). A novel and highly efficient method for the silylation of alcohols with hexamethyldisilazane (HMDS) catalyzed by recyclable sulfonic acid-functionalized ordered nanoporous silica. Tetrahedron Lett., 48(7), 1277-1280.

91. Jia, M., Seifert, A., Berger, M., Giegengack, H., Schulze, S., \& Thiel, W. R. (2004). Hybrid mesoporous materials with a uniform ligand distribution: synthesis, characterization, and application in epoxidation catalysis. Chem. Mater., 16(5), 877-882. 
92. Zhou, W., \& Fu, H. (2013). Mesoporous TiO2: preparation, doping, and as a composite for photocatalysis. ChemCatChem, 5(4), 885-894.

93. Zhang, H., Sun, J., Ma, D., Weinberg, G., Su, D. S., \& Bao, X. (2006). Engineered complex emulsion system: toward modulating the pore length and morphological architecture of mesoporous silicas. The J. of Phys. Chem. B, 110(51), 25908-25915.

94. Erathodiyil, N., Ooi, S., Seayad, A. M., Han, Y., Lee, S. S., \& Ying, J. Y. (2008). Palladium nanoclusters supported on propylurea-modified siliceous mesocellular foam for coupling and hydrogenation reactions. Chem.-A Europ. J., 14(10), 3118-3125.

95. Budarin, V., Luque, R., Macquarrie, D. J., \& Clark, J. H. (2007). Towards a bio-based industry: benign catalytic esterifications of succinic acid in the presence of water. Chem.-A Europ. J., 13(24), 69146919.

96. Dapurkar, S. E., Badamali, S. K., \& Selvam, P. (2001). Nanosized metal oxides in the mesopores of MCM-41 and MCM-48 silicates. Catal. Today, 68(1-3), 63-68.

97. Qu, F., Zhu, G., Huang, S., Li, S., \& Qiu, S. (2006). Effective controlled release of captopril by silylation of mesoporous MCM-41. Chemphyschem: a European journal of chemical physics and physical chemistry, 7(2), 400-406.

98. Brégeault, J. M., Vennat, M., Salles, L., Piquemal, J. Y., Mahha, Y., Briot, E., ... \& Thouvenot, R. (2006). From polyoxometalates to polyoxoperoxometalates and back again; potential applications. $J$. Molec. Catal. A: Chem., 250(1-2), 177-189.

99. Brunel, D., Cauvel, A., Di Renzo, F., Fajula, F., Fubini, B., Onida, B., \& Garrone, E. (2000). Preferential grafting of alkoxysilane coupling agents on the hydrophobic portion of the surface of micelle-templated silica. New J. Chem., 24(10), 807-813.

100. Innocenzi, P., Malfatti, L., Kidchob, T., \& Falcaro, P. (2009). Order- Disorder in self-assembled mesostructured silica films: a concepts review. Chem. Mater., 21(13), 2555-2564.

101. Fryxell, G. E., Mattigod, S. V., Lin, Y., Wu, H., Fiskum, S., Parker, K., ... \& Liu, J. (2007). Design and synthesis of self-assembled monolayers on mesoporous supports (SAMMS): The importance of ligand posture in functional nanomaterials. J. Mater. Chem., 17(28), 2863-2874.

102. Huq, R., Mercier, L., \& Kooyman, P. J. (2001). Incorporation of cyclodextrin into mesostructured silica. Chem. Mater., 13(12), 4512-4519.

103. Sun, J., \& Bao, X. (2008). Textural manipulation of mesoporous materials for hosting of metallic nanocatalysts. Chem.-A Europ. J., 14(25), 7478-7488.

104. Yuan, S., Sheng, Q., Zhang, J., Chen, F., Anpo, M., \& Zhang, Q. (2005). Synthesis of La3+ doped mesoporous titania with highly crystallized walls. Micropor. Mesopor. Mat., 79(1-3), 93-99.

105. Wirnsberger, G., Yang, P., Scott, B. J., Chmelka, B. F., \& Stucky, G. D. (2001). Mesostructured materials for optical applications: from low-k dielectrics to sensors and lasers. Spectrochimica Acta A, 57(10), 2049-2060.

106. Sheldon, R. A., Arends, I., \& Hanefeld, U. (2007). Green Chem. and catalysis. John Wiley \& Sons.

107. Sanchez, C., Lebeau, B., Ribot, F., \& In, M. (2000). Molecular design of sol-gel derived hybrid organicinorganic nanocomposites. J Sol.-Gel. Sci. Techn., 19(1-3), 31-38.

108. Boissière, C., Nicole, L., Gervais, C., Babonneau, F., Antonietti, M., Amenitsch, H., ... \& Grosso, D. (2006). Nanocrystalline mesoporous $\gamma$-alumina powders "UPMC1 Material" gathers thermal and chemical stability with high surface area. Chem. Mater., 18(22), 5238-5243.

109. Luo, Z., Poyraz, A. S., Kuo, C. H., Miao, R., Meng, Y., Chen, S. Y., ... \& Suib, S. L. (2014). Crystalline mixed phase (anatase/rutile) mesoporous titanium dioxides for visible light photocatalytic activity. Chem. Mater., 27(1), 6-17.

110. Qi, C., Zhu, Y. J., Zhao, X. Y., Lu, B. Q., Tang, Q. L., Zhao, J., \& Chen, F. (2013). Highly Stable Amorphous Calcium Phosphate Porous Nanospheres: Microwave-Assisted Rapid Synthesis Using ATP as Phosphorus Source and Stabilizer, and Their Application in Anticancer Drug Delivery. Chem.A Europ. J., 19(3), 981-987.

111. Qiu, H., \& Che, S. (2011). Chiral mesoporous silica: Chiral construction and imprinting via cooperative self-assembly of amphiphiles and silica precursors. Chem. Soc. Rev., 40(3), 1259-1268.

112. Sun, Y. L., Yang, B. J., Zhang, S. X. A., \& Yang, Y. W. (2012). Cucurbit [7] uril PseudorotaxaneBased Photoresponsive Supramolecular Nanovalve. Chem.-A Europ. J., 18(30), 9212-9216. 
113. Nicole, L., Rozes, L., \& Sanchez, C. (2010). Integrative approaches to hybrid multifunctional materials: from multidisciplinary research to applied technologies. Adv. Mater., 22(29), 3208-3214.

114. Brinker, C. J., \& Dunphy, D. R. (2006). Morphological control of surfactant-templated metal oxide films. Curr. Opin. Colloid In, 11(2-3), 126-132.

115. Schoedel, A., Wojtas, L., Kelley, S. P., Rogers, R. D., Eddaoudi, M., \& Zaworotko, M. J. (2011). Network Diversity through Decoration of Trigonal-Prismatic Nodes: Two-Step Crystal Engineering of Cationic Metal-Organic Materials. Angew. Chem-Ger Edit, 123(48), 11623-11626.

116. Yang, Z., Lu, Y., \& Yang, Z. (2009). Mesoporous materials: tunable structure, morphology and composition. Chem. Commun., (17), 2270-2277.

117. Yang, Q., Ma, S., Li, J., Xiao, F., \& Xiong, H. (2006). A water-compatible, highly active and reusable PEG-coated mesoporous silica-supported palladium complex and its application in Suzuki coupling reactions. Chem. Commun., (23), 2495-2497.

118. Kinnari, P., Mäkilä, E., Heikkilä, T., Salonen, J., Hirvonen, J., \& Santos, H. A. (2011). Comparison of mesoporous silicon and non-ordered mesoporous silica materials as drug carriers for itraconazole. Int. J. Pharm., 414(1-2), 148-156.

119. Bernardos, A., Aznar, E., Coll, C., Martínez-Mañez, R., Barat, J. M., Marcos, M. D., ... \& Soto, J. (2008). Controlled release of vitamin B2 using mesoporous materials functionalized with aminebearing gate-like scaffoldings. J. Control Release, 131(3), 181-189.

120. Luque, R., Budarin, V., Clark, J. H., \& Macquarrie, D. J. (2008). Glycerol transformations on polysaccharide derived mesoporous materials. Applied Catalysis B: Environmental, 82(3-4), 157-162.

121.Ciampi, S., Böcking, T., Kilian, K. A., Harper, J. B., \& Gooding, J. J. (2008). Click chemistry in mesoporous materials: functionalization of porous silicon rugate filters. Langmuir, 24(11), 5888-5892.

122. Hartmann, S., Brandhuber, D., \& Hüsing, N. (2007). Glycol-modified silanes: novel possibilities for the synthesis of hierarchically organized (hybrid) porous materials. Accounts Chem. Res., 40(9), 885894.

123. Fryxell, G. E. (2006). The synthesis of functional mesoporous materials. Inorg. Chem. Communications, 9(11), 1141-1150.

124. White, R. J., Brun, N., Budarin, V. L., Clark, J. H., \& Titirici, M. M. (2014). Always look on the "light" side of life: sustainable carbon aerogels. ChemSusChem, 7(3), 670-689.

125. Sinha, A. K., \& Suzuki, K. (2005). Three-Dimensional Mesoporous Chromium Oxide: A Highly Efficient Material for the Elimination of Volatile Organic Compounds. Angew. Chem. Int. Ent., 44(2), 271-273.

126. Shi, J. Y., Wang, C. A., Li, Z. J., Wang, Q., Zhang, Y., \& Wang, W. (2011). Heterogeneous organocatalysis at work: functionalization of hollow periodic mesoporous organosilica spheres with MacMillan catalyst. Chem.-A Europ. J., 17(22), 6206-6213.

127. Cheng, K., \& Landry, C. C. (2007). Diffusion-based deprotection in mesoporous materials: a strategy for differential functionalization of porous silica particles. J. Am. Chem. Soc., 129(31), 9674-9685.

128. Lindén, M., Schacht, S., Schüth, F., Steel, A., \& Unger, K. K. (1998). Recent advances in nano-and macroscale control of hexagonal, mesoporous materials. J. Porous Mat, 5(3-4), 177-193.

129. Li, G., Bhosale, S., Wang, T., Zhang, Y., Zhu, H., \& Fuhrhop, J. H. (2003). Gram-Scale Synthesis of Submicrometer-Long Polythiophene Wires in Mesoporous Silica Matrices. Angew. Chem. Int. Ent., 42(32), 3818-3821.

130. Ciriminna, R., Hesemann, P., Moreau, J. J., Carraro, M., Campestrini, S., \& Pagliaro, M. (2006). Aerobic oxidation of alcohols in carbon dioxide with silica-supported ionic liquids doped with perruthenate. Chem.-A Europ. J., 12(20), 5220-5224.

131.Gong, B., Peng, Q., Jur, J. S., Devine, C. K., Lee, K., \& Parsons, G. N. (2011). Sequential vapor infiltration of metal oxides into sacrificial polyester fibers: shape replication and controlled porosity of microporous/mesoporous oxide monoliths. Chem. Mater., 23(15), 3476-3485.

132. Miao, S., \& Shanks, B. H. (2009). Esterification of biomass pyrolysis model acids over sulfonic acidfunctionalized mesoporous silicas. Appl. Catal. B-Gen., 359(1-2), 113-120.

133.Zhou, Y., Tan, L. L., Li, Q. L., Qiu, X. L., Qi, A. D., Tao, Y., \& Yang, Y. W. (2014). Acetylcholinetriggered cargo release from supramolecular nanovalves based on different macrocyclic receptors. Chem.-A Europ. J., 20(11), 2998-3004. 
134. Jaramillo, T. F., Baeck, S. H., Kleiman-Shwarsctein, A., \& McFarland, E. W. (2004). Combinatorial electrochemical synthesis and screening of mesoporous $\mathrm{ZnO}$ for photocatalysis. Macromol. Rapid comm., 25(1), 297-301.

135. Wellmann, H., Rathousky, J., Wark, M., Zukal, A., \& Schulz-Ekloff, G. (2001). Formation of CdS nanoparticles within functionalized siliceous MCM-41. Micropor. Mesopor. Mat., 44, 419-425.

136. David, A. E., Wang, N. S., Yang, V. C., \& Yang, A. J. (2006). Chemically surface modified gel (CSMG): an excellent enzyme-immobilization matrix for industrial processes. J. Biotech., 125(3), 395407.

137.Zhou, Z., \& Hartmann, M. (2012). Recent progress in biocatalysis with enzymes immobilized on mesoporous hosts. Topics Catal., 55(16-18), 1081-1100.

138. Malvi, B., Sarkar, B. R., Pati, D., Mathew, R., Ajithkumar, T. G., \& Gupta, S. S. (2009). "Clickable" SBA-15 mesoporous materials: synthesis, characterization and their reaction with alkynes. $J$. Mater. Chem., 19(10), 1409-1416.

139. Kessel, S., Thomas, A., \& Börner, H. G. (2007). Mimicking biosilicification: programmed coassembly of peptide-polymer nanotapes and silica. Angew. Chem. Int. Ent., 46(47), 9023-9026.

140. Wang, Y., Bryan, C., Xu, H., Pohl, P., Yang, Y., \& Brinker, C. J. (2002). Interface chemistry of nanostructured materials: Ion adsorption on mesoporous alumina. J. Coll. Interf. Sci, 254(1), 23-30.

141. Gimenez, R., Lydon, D. P., \& Serrano, J. L. (2002). Metallomesogens: a promise or a fact?. Curr. Opin. Solid St. M. 6(6), 527-535.

142. Miao, R., Luo, Z., Zhong, W., Chen, S. Y., Jiang, T., Dutta, B., ... \& Suib, S. L. (2016). Mesoporous TiO 2 modified with carbon quantum dots as a high-performance visible light photocatalyst. App. Catal. B-Environ. 189, 26-38.

143.Zhang, L., Cha, D., \& Wang, P. (2012). Remotely controllable liquid marbles. Adv. Mater., 24(35), 4756-4760.

144. Masters, A. F., \& Maschmeyer, T. (2011). Zeolites-From curiosity to cornerstone. Micropor. Mesopor. Mat., 142(2-3), 423-438.

145. Mondal, J., Modak, A., \& Bhaumik, A. (2011). Highly efficient mesoporous base catalyzed Knoevenagel condensation of different aromatic aldehydes with malononitrile and subsequent noncatalytic Diels-Alder reactions. J. Molec. Catal. A: Chem., 335(1-2), 236-241.

146. Freund, C., Abrantes, M., \& Kühn, F. E. (2006). Monomeric cyclopentadiene molybdenum oxides and their carbonyl precursors as epoxidation catalysts. J. Organom. Chgem., 691(18), 3718-3729.

147. Choudary, B. M., Ramani, T., Maheswaran, H., Prashant, L., Ranganath, K. V. S., \& Kumar, K. V. (2006). Catalytic Asymmetric Epoxidation of Unfunctionalised Olefins using Silica, LDH and ResinSupported Sulfonato-Mn (salen) Complex. Adv. synth. Catalysis, 348(4-5), 493-498.

148. Galarneau, A., Villemot, F., Rodriguez, J., Fajula, F., \& Coasne, B. (2014). Validity of the t-plot method to assess microporosity in hierarchical micro/mesoporous materials. Langmuir, 30(44), 1326613274.

149. Balu, A. M., Budarin, V., Shuttleworth, P. S., Pfaltzgraff, L. A., Waldron, K., Luque, R., \& Clark, J. H. (2012). Valorisation of orange peel residues: waste to biochemicals and nanoporous materials. ChemSusChem, 5(9), 1694-1697.

150. Silva, S. S., Duarte, A. R. C., Carvalho, A. P., Mano, J. F., \& Reis, R. L. (2011). Green processing of porous chitin structures for biomedical applications combining ionic liquids and supercritical fluid technology. Acta biomater., 7(3), 1166-1172.

151. Nischang, I., Brüggemann, O., \& Teasdale, I. (2011). Facile, Single-Step Preparation of Versatile, High-Surface-Area, Hierarchically Structured Hybrid Materials. Angew. Chem. Int. Ent., 50(20), 45924596.

152. Vallet-Regí, M. (2010). Evolution of bioceramics within the field of biomaterials. Comptes Rendus Chimie, 13(1-2), 174-185.

153. Aznar, E., Coll, C., Marcos, M. D., Martínez-Máñez, R., Sancenon, F., Soto, J., ... \& Ruiz, E. (2009). Borate-driven gatelike scaffolding using mesoporous materials functionalised with saccharides. Chem.-A Europ. J., 15(28), 6877-6888.

154. Gracia, M. J., Losada, E., Luque, R., Campelo, J. M., Luna, D., Marinas, J. M., \& Romero, A. A. (2008). Activity of Gallium and Aluminum SBA-15 materials in the Friedel-Crafts alkylation of toluene with benzyl chloride and benzyl alcohol. Appl. Catal. B-Gen., 349(1-2), 148-155. 
155. Budarin, V. L., Clark, J. H., Luque, R., Macquarrie, D. J., Koutinas, A., \& Webb, C. (2007). Tunable mesoporous materials optimised for aqueous phase esterifications. Green Chem., 9(9), 992-995.

156. Corriu, R. J., Mehdi, A., Reyé, C., \& Thieuleux, C. (2003). Control of coordination chemistry in both the framework and the pore channels of mesoporous hybrid materials. New J. Chem., 27(6), 905-908.

157. White, R. J., Budarin, V. L., \& Clark, J. H. (2008). Tuneable Mesoporous Materials from $\alpha$-dPolysaccharides. ChemSusChem: Chemistry \& Sustainability Energy \& Materials, 1(5), 408-411.

158. Yoshitake, H. (2010). Design of functionalization and structural analysis of organically-modified siliceous oxides with periodic structures for the development of sorbents for hazardous substances. $J$. Mater. Chem., 20(22), 4537-4550.

159. Wang, D., Osmundsen, C. M., Taarning, E., \& Dumesic, J. A. (2013). Selective production of aromatics from alkylfurans over solid acid catalysts. ChemCatChem, 5(7), 2044-2050.

160. Mizoshita, N., Goto, Y., Kapoor, M. P., Shimada, T., Tani, T., \& Inagaki, S. (2009). Fluorescence emission from 2, 6-naphthylene-bridged mesoporous organosilicas with an amorphous or crystal-like framework. Chem.-A Europ. J., 15(1), 219-226.

161.Fischer, A., Makowski, P., Müller, J. O., Antonietti, M., Thomas, A., \& Goettmann, F. (2008). HighSurface-Area $\mathrm{TiO} 2$ and $\mathrm{TiN}$ as Catalysts for the C-C Coupling of Alcohols and Ketones. ChemSusChem: Chemistry \& Sustainability Energy \& Materials, 1(5), 444-449.

162. Goettmann, F., Moores, A., Boissière, C., Le Floch, P., \& Sanchez, C. (2005). A selective chemical sensor based on the plasmonic response of phosphinine-stabilized gold nanoparticles hosted on periodically organized mesoporous silica thin layers. Small, 1(6), 636-639.

163. Garcia-Bennett, A. E. (2011). Synthesis, toxicology and potential of ordered mesoporous materials in nanomedicine. Nanomedicine, 6(5), 867-877.

164. Su, D. S., Delgado, J. J., Liu, X., Wang, D., Schlögl, R., Wang, L., ... \& Xiao, F. S. (2009). Highly ordered mesoporous carbon as catalyst for oxidative dehydrogenation of ethylbenzene to styrene. Chem.-An Asian J., 4(7), 1108-1113.

165. Guo, W., Wang, J., Lee, S. J., Dong, F., Park, S. S., \& Ha, C. S. (2010). A general pH-responsive supramolecular nanovalve based on mesoporous organosilica hollow nanospheres. Chem.-A Europ. J., 16(29), 8641-8646.

166. White, R. J., Budarin, V. L., \& Clark, J. H. (2010). Pectin-Derived Porous Materials. Chem.-A Europ. $J ., 16(4), 1326-1335$.

167. Nowotny, M., Pedersen, L. N., Hanefeld, U., \& Maschmeyer, T. (2002). Increasing the ketone selectivity of the cobalt-catalyzed radical chain oxidation of cyclohexane. Chem.-A Europ. J., 8(16), 3724-3731.

168. Campbell, A. S., Dong, C., Meng, F., Hardinger, J., Perhinschi, G., Wu, N., \& Dinu, C. Z. (2014). Enzyme catalytic efficiency: a function of bio-nano interface reactions. ACS applied materials \& interfaces, 6(8), 5393-5403.

169. Ng, A., Ciampi, S., James, M., Harper, J. B., \& Gooding, J. J. (2009). Comparing the reactivity of alkynes and alkenes on silicon (100) surfaces. Langmuir, 25(24), 13934-13941.

170. Valentin, R., Molvinger, K., Viton, C., Domard, A., \& Quignard, F. (2005). From hydrocolloids to high specific surface area porous supports for catalysis. Biomacromolecules, 6(5), 2785-2792.

171. Kurth, D. G., Fromm, K. M., \& Lehn, J. M. (2001). Hydrogen-Bonding and Metal-Ion-Mediated SelfAssembly of a Nanoporous Crystal Lattice. Europ. J. of Inorg. Chem., 2001(6), 1523-1526.

172. Bariana, M., Aw, M. S., Kurkuri, M., \& Losic, D. (2013). Tuning drug loading and release properties of diatom silica microparticles by surface modifications. Int. J. Pharm., 443(1-2), 230-241.

173. Yang, D., Paul, B., Xu, W., Yuan, Y., Liu, E., Ke, X., ... \& Zhu, H. (2010). Alumina nanofibers grafted with functional groups: a new design in efficient sorbents for removal of toxic contaminants from water. Water research, 44(3), 741-750.

174.Pérez-Quintanilla, D., Gómez-Ruiz, S., Žižak, Ž., Sierra, I., Prashar, S., del Hierro, I., ... \& Kaluđerović, G. N. (2009). A new generation of anticancer drugs: mesoporous materials modified with titanocene complexes. Chem.-A Europ. J., 15(22), 5588-5597.

175. Dufaud, V., Beauchesne, F., \& Bonneviot, L. (2005). Organometallic chemistry inside the pore walls of mesostructured silica materials. Angew. Chem. Int. Ent., 44(22), 3475-3477.

176. An, K., Alayoglu, S., Ewers, T., \& Somorjai, G. A. (2012). Colloid chemistry of nanocatalysts: A molecular view. J. Coll. Interf. Sci, 373(1), 1-13. 
177. Gao, Z., Feng, Y., Cui, F., Hua, Z., Zhou, J., Zhu, Y., \& Shi, J. (2011). Pd-loaded superparamagnetic mesoporous $\mathrm{NiFe} 2 \mathrm{O} 4$ as a highly active and magnetically separable catalyst for Suzuki and Heck reactions. J. Mol. Catal. A-Chem., 336(1-2), 51-57.

178. González, B., Colilla, M., de Laorden, C. L., \& Vallet-Regí, M. (2009). A novel synthetic strategy for covalently bonding dendrimers to ordered mesoporous silica: potential drug delivery applications. $J$. Mater. Chem., 19(47), 9012-9024.

179. Grosso, D., Babonneau, F., Sanchez, C., de AA Soler-Illia, G. J., Crepaldi, E. L., Albouy, P. A., ... \& Brunet-Bruneau, A. (2003). A first insight in the mechanisms involved in the self-assembly of 2Dhexagonal templated $\mathrm{SiO} 2$ and $\mathrm{TiO} 2$ mesostructured films during dip-coating. J Sol.-Gel. Sci. Techn., 26(1-3), 561-565.

180. Soler-Illia, G. J. D. A., Rozes, L., Boggiano, M. K., Sanchez, C., Turrin, C. O., Caminade, A. M., \& Majoral, J. P. (2000). New mesotextured hybrid materials made from assemblies of dendrimers and titanium (IV)-oxo-organo clusters. Angew. Chem-Ger Edit, 112(23), 4419-4424.

181.Du, M., Zhu, P., Yan, X., Su, Y., Song, W., \& Li, J. (2011). Honeycomb self-assembled peptide scaffolds by the breath figure method. Chem.-A Europ. J., 17(15), 4238-4245.

182.Liu, J., Du, X., \& Zhang, X. (2011). Enzyme-Inspired Controlled Release of Cucurbit [7] uril Nanovalves by Using Magnetic Mesoporous Silica. Chem.-A Europ. J., 17(3), 810-815.

183. Anurova, N. A., Blatov, V. A., Ilyushin, G. D., \& Proserpio, D. M. (2010). Natural tilings for zeolitetype frameworks. The J. Physic. Chem. C, 114(22), 10160-10170.

184. Kilian, K. A., Böcking, T., Gaus, K., \& Gooding, J. J. (2008). Introducing distinctly different chemical functionalities onto the internal and external surfaces of mesoporous materials. Angew. Chem-Ger Edit, 120(14), 2737-2739.

185. Verma, S., Nandi, M., Modak, A., Jain, S. L., \& Bhaumik, A. (2011). Novel Organic-Inorganic Hybrid Mesoporous Silica Supported Oxo-Vanadium Schiff Base for Selective Oxidation of Alcohols. Adv. synth. Catalysis, 353(11-12), 1897-1902.

186. Muñoz-Espí, R., Weiss, C. K., \& Landfester, K. (2012). Inorganic nanoparticles prepared in miniemulsion. Curr. Opin. Colloid In, 17(4), 212-224.

187. Saini, V. K., Andrade, M., Pinto, M. L., Carvalho, A. P., \& Pires, J. (2010). How the adsorption properties get changed when going from SBA-15 to its CMK-3 carbon replica. Sep. Purfic. Technol., 75(3), 366-376.

188. Kuschel, A., Sievers, H., \& Polarz, S. (2008). Amino acid silica hybrid materials with mesoporous structure and enantiopure surfaces. Angew. Chem. Int. Ent., 47(49), 9513-9517.

189. Deshpande, A. S., Burgert, I., \& Paris, O. (2006). Hierarchically Structured Ceramics by HighPrecision Nanoparticle Casting of Wood. Small, 2(8-9), 994-998.

190. Agirrezabal-Telleria, I., Requies, J., Güemez, M. B., \& Arias, P. L. (2014). Dehydration of d-xylose to furfural using selective and hydrothermally stable arenesulfonic SBA-15 catalysts. App. Catal. BEnviron. 145, 34-42.

191. Thommes, M., Mitchell, S., \& Pérez-Ramírez, J. (2012). Surface and pore structure assessment of hierarchical MFI zeolites by advanced water and argon sorption studies. The J. of Physic. Chem. $C, 116(35), 18816-18823$.

192.Park, S. S., \& Ha, C. S. (2006). Organic-inorganic hybrid mesoporous silicas: functionalization, pore size, and morphology control. The Chem. Rec., 6(1), 32-42.

193. Wei, Y., Xu, J., Feng, Q., Lin, M., Dong, H., Zhang, W. J., \& Wang, C. (2001). A novel method for enzyme immobilization: direct encapsulation of acid phosphatase in nanoporous silica host materials. J. Nanosci. Nanotechno., 1(1), 83-93.

194. Hartmann, P., Brezesinski, T., Sann, J., Lotnyk, A., Eufinger, J. P., Kienle, L., \& Janek, J. (2013). Defect chemistry of oxide nanomaterials with high surface area: ordered mesoporous thin films of the oxygen storage catalyst CeO2-ZrO2. ACS nano, 7(4), 2999-3013.

195. Bohström, Z., Rico-Lattes, I., \& Holmberg, K. (2010). Oxidation of cyclohexene into adipic acid in aqueous dispersions of mesoporous oxides with built-in catalytical sites. Green Chem., 12(10), 18611869.

196.Kim, Y., Kim, C., \& Yi, J. (2004). Synthesis of tailored porous alumina with a bimodal pore size distribution. Mater. Res. Bull., 39(13), 2103-2112. 
197. Sinha, A. K., \& Suzuki, K. (2007). Novel mesoporous chromium oxide for VOCs elimination. App. Catal. B-Environ. 70(1-4), 417-422.

198. Michailovski, A., \& Patzke, G. R. (2006). Hydrothermal synthesis of molybdenum oxide based materials: Strategy and structural chemistry. Chem.-A Europ. J., 12(36), 9122-9134.

199. Bhoware, S. S., Shylesh, S., Kamble, K. R., \& Singh, A. P. (2006). Cobalt-containing hexagonal mesoporous molecular sieves (Co-HMS): Synthesis, characterization and catalytic activity in the oxidation reaction of ethylbenzene. J. Mol. Catal. A-Chem., 255(1-2), 123-130.

200. Walcarius, A., Delacote, C., \& Sayen, S. (2004). Electrochemical probing of mass transfer rates in mesoporous silica-based organic-inorganic hybrids. Electrochimica acta, 49(22-23), 3775-3783.

201. Sayen, S., Etienne, M., Bessière, J., \& Walcarius, A. (2002). Tuning the Sensitivity of Electrodes Modified with an Organic-Inorganic Hybrid by Tailoring the Structure of the Nanocomposite Material. Electroanal, 14(21), 1521-1525.

202. Garcia-Martinez, J., Xiao, C., Cychosz, K. A., Li, K., Wan, W., Zou, X., \& Thommes, M. (2014). Evidence of intracrystalline mesostructured porosity in zeolites by advanced gas sorption, electron tomography and rotation electron diffraction. Chem CatChem, 6(11), 3110-3115.

203. Esfahani, H., Tavasoli, K., \& Jabbarzadeh, A. (2019). Big data and social media: A scientometrics analysis. Int. J. Data Net. Sci, 3(3), 145-164.

204. Salimi, D., Tavasoli, K., Gilani, E., Jouyandeh, M., \& Sadjadi, S. (2019). The impact of social media on marketing using bibliometrics analysis. Int. J. Data Net. Sci, 3(3), 165-184.

205. Alavi, S., Mehdinezhad, I., \& Kahshidinia, B. (2019). A trend study on the impact of social media on advertisement. Int. J. Data Net. Sci, 3(3), 185-200.

206. Gilani, E., Salimia, D., Jouyandeh, M., Tavasoli, K., \& Wong, W. (2019). A trend study on the impact of social media in decision making. Int. J. Data Net. Sci, 3(3), 201-222.

207.Pourkhani, A., Abdipour, K., Baher, B., \& Moslehpour, M. (2019). The impact of social media in business growth and performance: A scientometrics analysis. Int. J. Data Net. Sci, 3(3), 223-244.

208. Tayebi, S., Manesh, S., Khalili, M., \& Sadi-Nezhad, S. (2019). The role of information systems in communication through social media. Int. J. Data Net. Sci, 3(3), 245-268.

209. Javid, E., Nazari, M., \& Ghaeli, M. (2019). Social media and e-commerce: A scientometrics analysis. Int. J. Data Net. Sci., 3(3), 269-290.

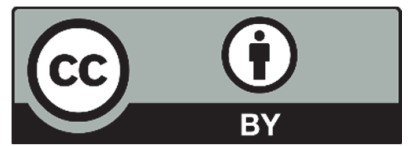

(C) 2019 by the authors; licensee Growing Science, Canada. This is an open access article distributed under the terms and conditions of the Creative Commons Attribution (CC-BY) license (http://creativecommons.org/licenses/by/4.0/). 